\title{
Atomic force microscopy of bacteria from periodontal subgingival biofilm: Preliminary study results
}

\author{
Francesco Germano ${ }^{1}$, Ennio Bramanti ${ }^{2}$, Claudio Arcuri ${ }^{1}$, Francesco Cecchetti ${ }^{1}$, Marco Cicciù ${ }^{3}$
}

Correspondence: Dr. Marco Cicciù

Email: acromarco@yahoo.it

\author{
'Odontostomatology Department, University of Roma \\ Torvergata School of Dentistry, RM, Italy, \\ ${ }^{2}$ Odontostomatology Department, University of \\ Messina School of Dentistry, ME, Italy, \\ ${ }^{3}$ Human Pathology Department, University of Messina \\ School of Dentistry, ME, Italy
}

\section{ABSTRACT}

Objective: Atomic force microscope (AFM) is a technology that allows analysis of the nanoscale morphology of bacteria within biofilm and provides details that may be better useful for understanding the role of bacterial interactions in the periodontal disease. Material and Methods: Five patients with periodontal $\geq 5 \mathrm{~mm}$ pockets diagnosed as generalized periodontitis and five patients with slight gingivitis were selected for the investigation. Bacteria biofilms were collected and morphologically investigated by AFM application. Results: The investigation revealed how periodontitis bacteria are characterized by specific morphologic features of the cell wall. The major representative species of bacteria causing periodontal diseases have been reproduced by a three-dimensional reconstruction showing the bacteria surface details. Conclusions: The presence of complex glycocalyx structures, bacteriophage-like vesicles, spirochetes (classic and cystic morphology) and bacterial co-aggregation has been identified by the AFM analysis. The results suggest that AFM is a reliable technique for studying bacterial morphology and for examining microbial interactions in dental plaque.

Key words: Atomic force microscopy, biofilm, microbiology, periodontitis

\section{INTRODUCTION}

Periodontitis is a multifactorial disease caused by bacterial biofilm living on tooth surface and sockets. Researchers have attempted to identify the species responsible for the different forms of periodontitis with increasingly molecular investigation methods of bacterial culture. ${ }^{[1,2]}$ This hope has clashed with the inability to cultivate the various species related with periodontal disease. ${ }^{[3]}$ Moreover, high difficulty is related to the identification of complex multispecies interrelationships of oral biofilm. The bacteria presented at the oral cavity can be morphologically divided into six shape classes: Coccoid cell, straight rods, fusiform, filament, flagellated and spirochete. Moreover, the health of the oral cavity is related with the presence of several microorganisms that may influence the oral pathology onset. ${ }^{[4]}$ The great morphological heterogeneity of periodontal bacterial species corresponds to an even bigger genetic variability as demonstrated by techniques of recombinant DNA and studies of molecular phylogenetics related. ${ }^{[5]}$

Several researches demonstrated how periodontal diseases are influenced by the presence of bacterial species in the biofilms covering the dental surfaces. ${ }^{[6]}$ The microbial composition of dental biofilms is based on studies of samples from mature dental plaque. Numerous investigations revealed how flagellated bacteria and spirochetes constitute the most common sub-gingival species in chronic periodontitis. In the recent years, many researchers started analyses of interspecies interaction of bacterial biofilms, adding more individual species studies. ${ }^{[7,8]}$

\footnotetext{
How to cite this article: Germano F, Bramanti E, Arcuri C, Cecchetti F, Cicciù M. Atomic force microscopy of bacteria from periodontal subgingival biofilm: Preliminary study results. Eur J Dent 2013;7:152-8. 
Morphological researches of bacteria discovered new methods of detection by using optical microscopy in dark fields, phase contrast, scanning with transmission electron microscopy and confocal laser scanning microscopy. ${ }^{[9-11]}$ The introduction of atomic force microscope (AFM) in microbiological studies helped researchers with a new device for the analysis of the bacterial morphology. This device can finally investigate the three-dimensional structure of the outer membrane, glycocalyx covering the bacteria and bacteriophage. ${ }^{[12,13]}$ One of the advantages of AFM is the easy sample preparation and the achievable high resolution. This condition makes it a promising tool in periodontal research. This device does not use color application for underlining bacteria cytological features. In this way, it is possible to have detailed results with lower cost and reduced investigation time.

The specific morphological characteristics of the bacterial cell wall could be useful in identifying specific virulent factors associated with aggressive periodontitis. To date, this method has not yet been used to study the morphological characteristics of periodontal bacteria. The aim of this preliminary study is to use AMF for describing the morphology of some periodontal bacterial species in patients with gingivitis and periodontitis.

\section{MATERIALS AND METHODS}

\section{Biofilm sample selection}

Bacteria biofilms were sampled from five patients with clinical periodontal $\geq 5 \mathrm{~mm}$ pockets diagnosed by probe as generalized aggressive periodontitis and from five patients with slight gingivitis with a deep probing ranging from $3 \mathrm{~mm}$ to $5 \mathrm{~mm}$. All the selected patients undersigned an informed consent explaining all the procedures and the research project. The Ethical Committee of the San Giovanni Calibita Fatebenefratelli Hospital Rome approved the research. Patients with systemic pathologic condition, treated with no steroidal anti-inflammatory drug, or assuming corticosteroid drugs or antibiotics in the last 6 months were not included in the investigation. Ten plaque biofilm samples were collected from each investigated patient.

\section{Slide preparations}

Biofilm samples were collected with a sterile curette from the gingival sulcus of the patients with gingivitis and from the deep sockets area of the patients with periodontitis after carefully removing the supragingival plaque. The biofilms were dispersed in $200 \mu$ l of sterile distilled water using a sterile syringe (needle 10 Gauge) with gentle movements of suction to obtain a turbid solution without visible bacterial aggregates. Twenty microliters of this solution was then smeared onto sterile slides, allowed to dry and stored at room temperature until examination within 2 days. Two slides for each sample were prepared.

\section{Atomic force microscopy}

Images were taken with an AFM SolverPro-M (NT-MDT, Moscow-Russia) ${ }^{\circledR}$ in semi-contact and phase-mode, scanning-by-sample, equipped with ETALON HA_NC/15 probes. All images were acquired at 1024 lines. Image analysis was performed using the free software Gwyddion. ${ }^{\circledR}$

\section{RESULTS}

All biofilms obtained from slight gingivitis patients were associated with predominant bacterial species of coccoid or straight rod bacteria [Figure 1a and b]. AFM acquisition showed a three-dimensional image, which was useful in clarifying the different shapes and heights of the bacteria and the vesicles [Figure 1c]. The three-dimensional image represents cocci bacteria with different morphology. The image classifies a dividing coccus (as indicated by the arrow) and three single cocci bacteria with a flattened surface, which probably are dead bacteria or more likely cell wall-deficient bacteria. ${ }^{[14]}$ Diplococcus bacteria with some vesicles attached to the outer bacteria membrane can be highlighted by this image too [Figure $1 \mathrm{~b}$ and $\mathrm{c}$ center of the image]. The size of these vesicles was about $100 \mathrm{~nm}$. The presence of the vesicles is observed around the bacterial aggregate and in the bottom-left part of the image as well. Two kind of vesicles were seen; a small one measuring up to $50 \mathrm{~nm}$ and a large one measuring around $100 \mathrm{~nm}$. These vesicles were likely to have been released from neighboring bacteria. ${ }^{[12-15]}$

In biofilm samples coming from patients with periodontitis, we observed that the species mainly represented are spirochetes, flagellated forms and filaments, while coccoid and straight rod species are less represented [Figure 2a]. The analyzed spirochetes show different features and size. It can be underlined a group of small (0.2-0.3 $\mu \mathrm{m}$ wide, up to $10 \mu \mathrm{m}$ large), intermediate $(0.3-0.4 \mu \mathrm{m}$ wide, up to $15 \mu \mathrm{m}$ large) and large ( $0.5 \mu \mathrm{m}$ or wider, up to $20 \mu \mathrm{m}$ large) [Figure 2a-c]. ${ }^{[6,15]}$ High magnification of the small spirochete, acquired in "phase mode," shows 


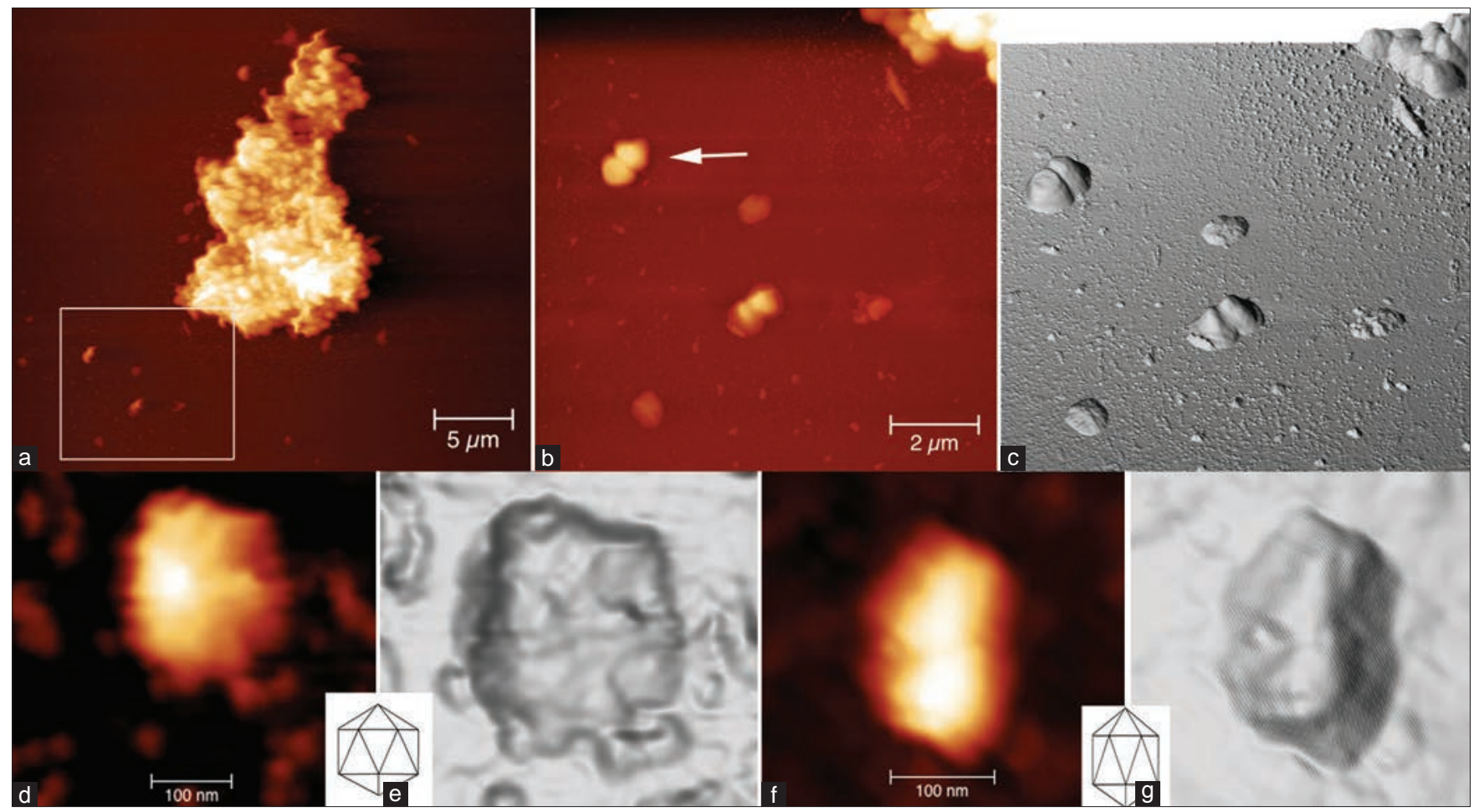

Figure 1: (a) A cluster of bacteria is surrounded by many cocci and a straight rod bacteria (arrow); white box shows the section enlarged in (b) From the atomic force microscopy (AFM) data present in (b), we obtained a three-dimensional reconstruction (c) Cocci with different morphology and staging can be observed ( $b$ and $c$ ). We observed a dividing coccus as indicated by the arrow and three single cocci with a flattened surface (see text). Two kinds of vesicles: Small vesicle measuring up to $50 \mathrm{~nm}$ and large vesicle measuring around $100 \mathrm{~nm}$, could be seen (b and c). On high magnification ( $\mathrm{d}$ and $\mathrm{f}$ ) along with three-dimensional reconstructions (e and $\mathrm{g}$ ) of two large vesicles, their morphology was found to be compatible with icosahedral forms of the bacteriophage heads as clarified by the comparison between two icosahedrons' drawings (showed in insets). Heights of the images are 0-190 $\mathrm{nm}(\mathrm{a}), 0-170 \mathrm{~nm}(\mathrm{~b}$ and c) and 0-30 $\mathrm{nm}$ ( $\mathrm{d}$ and f). Measure bars as shown

a knoblike structure in the upper end [Figure 2d]. Phase-mode acquisition is the device that is particularly useful for discerning fine details of the outer membrane, like roughness, vesicle, fimbria or pili. This investigation also gives an image about a cystic form of a spirochete with a spiral body, coiled and surrounded by a thin membrane [Figure 2e]. ${ }^{[16,17]}$ Another type of evidenced bacteria is a large spirochete with the outer membrane partially dissolved, axial fibril emerging from the left head and a large vesicle emerging from the center of the body that appears to have a granular content [Figure 2f]. Moreover, a spirochete with several vesicles emerging from the body is underlined too [Figure 2d]. Several published papers state that cystic and granular forms of spirochetes do not represent an aberrant form but are instead part of their complex life cycle. ${ }^{[18]}$ Recent literary reports affirm that bacterial species related to severe periodontitis are flagellated rods too. A curved rod with flagella emerging from the concave side of the cell wall is still presented by Figures $3 a, 3 b$ and 3c. The investigation and a three dimensional reconstruction of the same image is also underlined by Figures 3d, 3e and 3f. A straight rod with a septum and flagella emerging from both sides and a small coccus is also visible near the rod, as highlighted in Figures $3 \mathrm{~b}$ and e. A dividing curved rod and a septum are clearly visible in the middle of the body with flagella emerging from the concave cell wall in Figures $3 \mathrm{c}$ and $\mathrm{f}$. The flagella are separated into two branches and a fine net of vesicles is revealed between the diverging flagella.

The other cells normally presented in the oral biofilms are leucocyte type (mainly polymorphonucleate [PMN] and macrophage). A round cell compatible with a leucocyte (PMN), as suggested by the size, around $13 \mu \mathrm{m}$ of diameter and by morphology is shown in the Figure 4a. The flattened cell's morphology is probably due to sample preparation. Inside this cell there is a non-homogeneous zone that could be the nucleus (not demonstrated). Moreover, bacteria with different morphology (flagellated rod, fusiform, coccoid) in contact with the leucocyte have been discovered by those samples. It is possible to evidence a round cell (leukocyte like) in Figures $4 \mathrm{~b}$ and c. The diameter of those cells is about $10 \mu \mathrm{m}$ and, inside, the presence of some vesicles is underlined in a non-homogeneous 


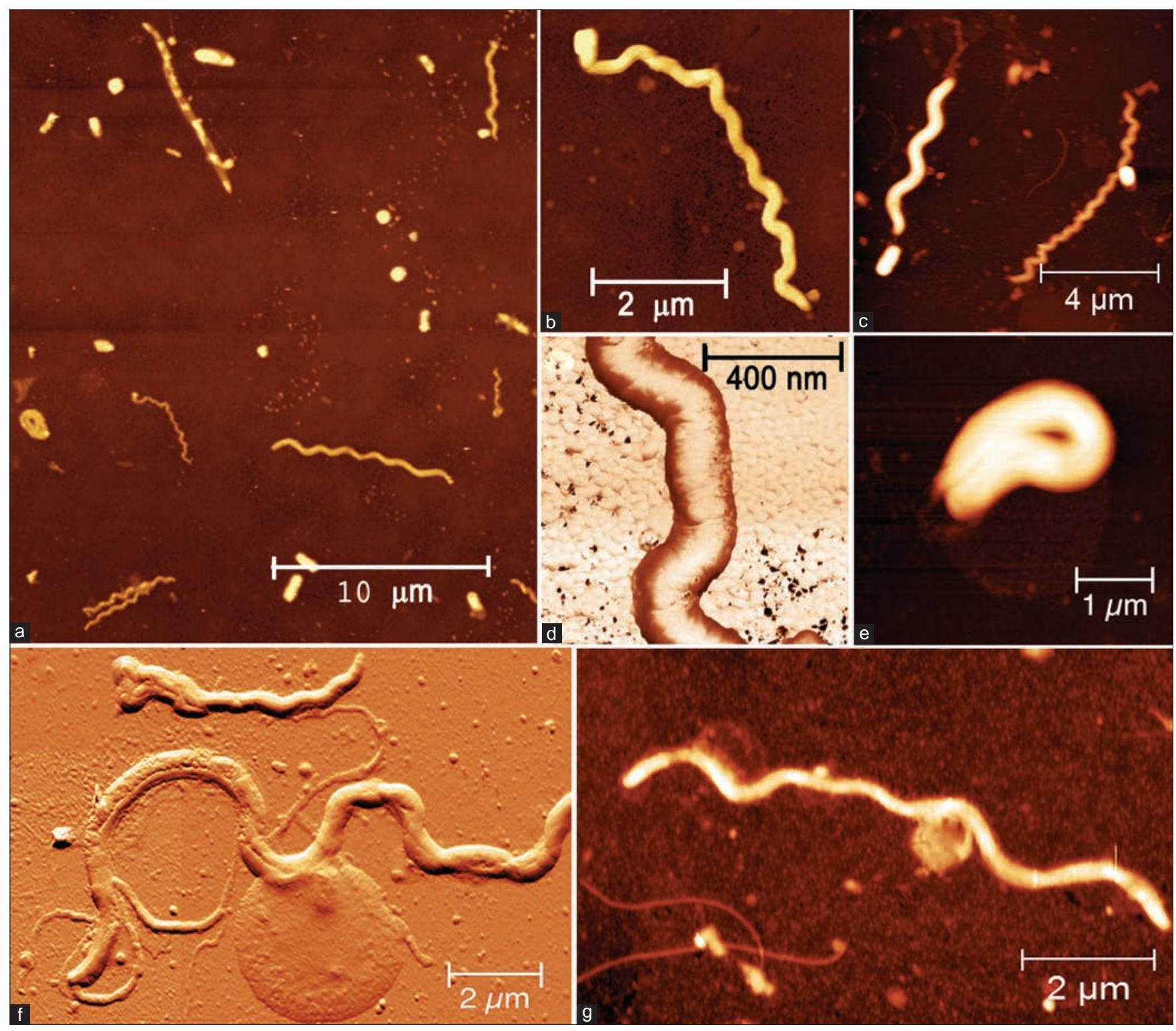

Figure 2: (a) All the major bacterial species are present: Spirochetes (small, intermediate, large and cystic), flagellated forms, filaments, coccoid and straight rod, (b) Small spirochete $(0.2 \mu \mathrm{m}$ wide, $10 \mu \mathrm{m}$ large) with a knoblike structure in the upper end, (d) High magnification of a thread of a small spirochete (box b) acquired in the "phase mode," (c) Small spirochete (on the right) and intermediate spirochete (on the left), (e) Cystic form of a spirochete in which the spiral body appears coiled and is surrounded by a thin membrane, (f) Large spirochete with outer membrane partially dissolved, axial fibril emerging from the left head and a large vesicle emerging from the center of the body, which appears to have a granular content, (g) Spirochete with some vesicle emerging from the body. See text for detail. Heights of the images are 0-400 $\mathrm{nm}(\mathrm{a}), 0-170 \mathrm{~nm}$ (b, $\mathrm{f}$ and $\mathrm{g}), 0-250 \mathrm{~nm}(\mathrm{c}), 0-130 \mathrm{~nm}(\mathrm{~d})$, and 0-200 $\mathrm{nm}(\mathrm{e})$, Measure bars as shown

zone-area. A fusiform bacteria adherent to the cell membrane, which could be a phagocytised bacterium, is presented as well. However, the presence of a cell overlapping, due to the sample preparation, cannot be excluded. A complex biofilm coming from a periodontitis patient is shown in Figure $4 d$, where filamentous bacteria and spirochetes are surrounded by a complex glycocalyx. The magnification of the figure with its three-dimensional reconstruction is shown, respectively, in Figure $4 \mathrm{e}$ and $\mathrm{f}$. The complexity of the glycocalyx composed by a fine net of different vesicles can be observed too.

\section{DISCUSSION}

The observation of large vesicles at high magnification [Figure 1d and f], supported by the three-dimensional reconstructions [Figure 1e and g], allowed to underline how the bacterial morphology is compatible with icosahedral forms of bacteriophage head, as clarified by the comparison with the inset of two icosahedron drawings. ${ }^{[13-15]}$

More than 600 microbiological species have been recorded in the human mouth. The oral cavity is 


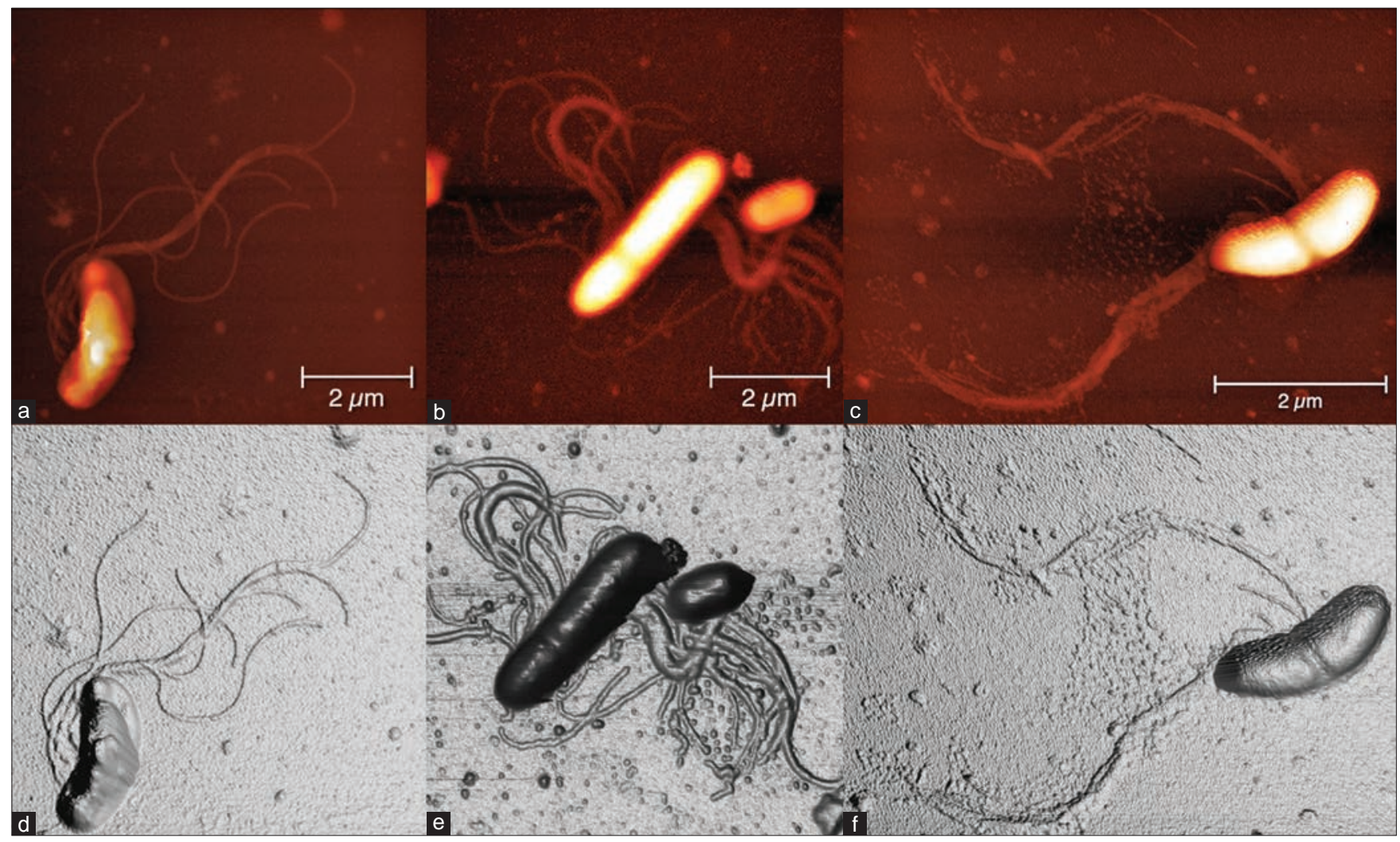

Figure 3: Samples from patients with periodontitis. (a) Curved rod with flagella emerging from the concave side of the cell wall. (d) Three-dimensional reconstruction of (a), (b) Straight rod with a septum and flagella emerging from both sides. A small coccus is present near the flagellated rod. (e) Three-dimensional reconstruction of (b). (c) A dividing curved rod is shown; a septum is clearly visible in the middle of the body with flagella emerging from the concave cell wall. The flagella are separated into two branches and a fine net of vesicle is present between the diverging -flagella. (f) Three-dimensional reconstruction of (c) Heights of the images are 0-190 nm (a-f). Measure bars as shown

also home to microbial communities with numerous implications for human health and disease. Chronic periodontitis is one of the most common inflammatory conditions worldwide, and it can be related with bacterial community structures that are distinct from those of health. The saprophyte microbiota of an individual is included in the range between about 100 species. A wide variety of oral cavity sites are differently colonized. The number of microbial groups on and within the human body varies between subjects. Individual diversities have been recorded in several studies for the healthy intestinal tract and oral cavity. ${ }^{[19]}$ In contrast, knowledge about the subjects individual differences in the healthy human mouth microbiota and the uniqueness of the oral microbiota compared with other microbial communities in our bodies is still somewhat sparse. ${ }^{[11,20]}$

Supragingival and subgingival plaque form through sequential and specific adhesive interactions and result in a favorable climax community. ${ }_{[5,7,15]}$

This study describes for the first time the application of an atomic force microscope in bacteria extracted from subgingival plaque biofilms. The application of this device helps to increase the knowledge of the research of periodontal bacterial species. Periodontitis is a multifactorial disease that involves the hard and soft tissue, microbial colonization inflammatory responses and consequent immune responses. The variety of the local tissue elements, including bacteria and their products associated with the host response mechanisms components, has altered the effectiveness of protective functions in the host tissues. ${ }^{[15,21]}$

The mechanisms of control of periodontal pathogens are much debated. The inhibitory activity displayed against periodontal pathogens seems to be connected with their production of acid. The possibility of identifying the bacterial structure features may help clinicians in recognizing the type of disease and performing the right treatment for the presented bacteria. ${ }^{[15,17]}$ This study is aimed at the characterization of bacterial morphotype, at the identification of specific morphological characteristics of bacterial cell wall and membrane structures like vesicles. Another goal of the study is related with the identification of bacteriophages that could be useful to the research 

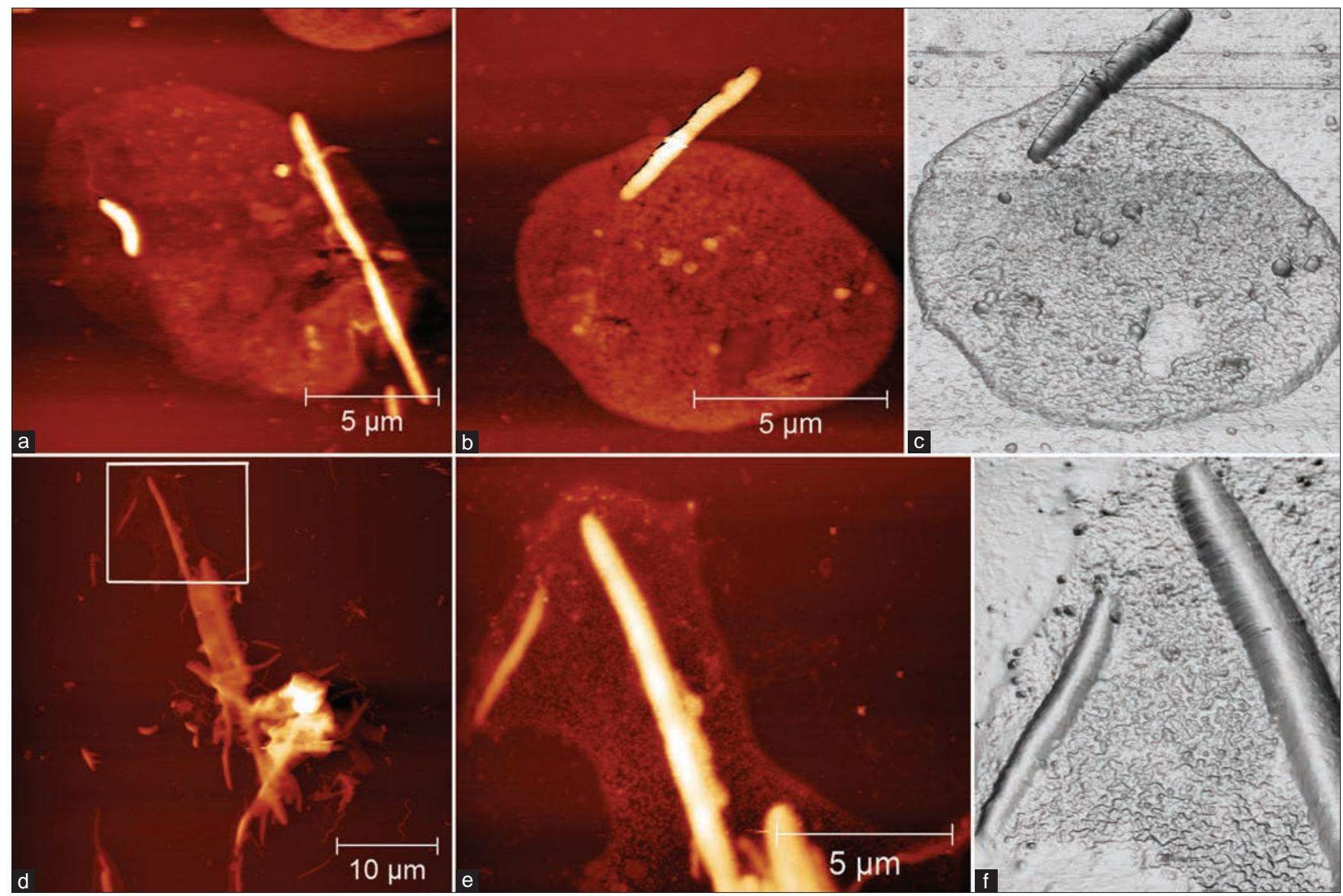

Figure 4: (a) Round cell that measures around $13 \mu \mathrm{m}$ in diameter, appearing flattened due to sample preparation. Inside this cell, some non-homogeneous zones are present. The size and morphology of this cell are compatible with a leukocyte, probably a polymorphonucleate (PMN). Bacteria with different morphology (flagellated rod, fusiform, coccoid) can be seen in contact with the leucocyte. (bB) Three-dimensional reconstruction and (c) round cell measuring around $10 \mu \mathrm{m}$ in diameter (compatible with PMN leukocyte). Inside this cell, some vesicle and some non-homogeneous zone (nucleus) can be seen. A fusiform bacteria adherent to the cell membrane is present, which may represent bacteria that have been phagocytized or superimposed to the cell due to sample preparation. (d) Complex biofilm coming from a periodontitis patient. Some filaments along with spirochete are surrounded by a glycocalyx. A magnification of (d) with its three-dimensional reconstruction are shown, respectively, in (e) and (f). Heights of the images are 0-190 $\mathrm{nm}(\mathrm{a}-\mathrm{f})$ and 0-250 $\mathrm{nm}(\mathrm{d})$. Measure bars as shown

of periodontal disease bacteria. Bacterial glycocalyx gives protection against such environmental threats as antibiotics, antibodies and white blood cells. ${ }^{[21]}$ For this reason, a research involving the glycocalyx features may provide help in the research of the periodontal disease virulent action.

Bacteriophages are associated with aggressive forms of periodontitis and are responsible for the spreading of the virulence factor during the outbreak of aggressive periodontitis. ${ }^{[22]}$ Sample preparation procedures for AFM allow preserving the fine glycocalyx structures and bacteriophages. That could be used to discern between several pathologic biofilms. AFM action may help in the analysis and evaluation of complex glycocalyx structures, bacteriophage-like vesicles, spirochetes (classic and cystic morphology) and bacterial co-aggregation.
Unlike the other methods currently used for three-dimensional analysis, such as scanning electron microscopy (which requires fixation, dehydration, air drying and metal coating), AFM has a straightforward biofilm slide preparation attainable by air-drying. The purpose of this pilot study was to reveal the advantages of AFM in the morphological study of microorganisms and their interactions within subgingival biofilm in different periodontal conditions.

The advantages of AFM are related with the possibility of obtaining the structure of biomolecules or bio-surfaces with molecular (or even submolecular) resolution; moreover, the investigators can measure local chemical, physical and mechanical properties of bacteria surfaces (charge density, adhesion, rigidity, viscosity, elasticity) and intermolecular forces within a spatial resolution below $1 \mathrm{~nm} \cdot{ }^{[23]}$ 
However, future studies are needed to associate morphological characteristics of biofilm to different periodontal diseases' seriousness in order to be able to distinguish among them. However, those preliminary results offer researchers the opportunity to better understand the bacterial features by tridimensional investigation.

\section{REFERENCES}

1. Gursoy UK. Periodontal bacteria and epithelial cell interactions: Role of bacterial proteins. Eur J Dent 2008;2:231-2.

2. van Winkelhoff AJ, Loos BG, van der Reijden WA, van der Velden U. Porphyromonas gingivalis, bacteroides forsythus and other putative periodontal pathogens in subjects with and without periodontal destruction. J Clin Periodontol 2002;29:1023-8.

3. Loesche WJ, Lopatin DE, Stoll J, van Poperin N, Hujoel PP. Comparison of various detection methods for periodontopathic bacteria: Can culture be considered the primary reference standard? J Clin Microbiol 1992;30:418-26.

4. Akin L, Herford AS, Cicciù M. Oral presentation of disseminated histoplasmosis: A case report and literature review. J Oral Maxillofac Surg 2011;69:535-41

5. Hugenholtz P, Pace NR. Identifying microbial diversity in the natural environment: A molecular phylogenetic approach. Trends Biotechnol 1996;14:190-7.

6. Mohamed Saber SE, El-Hady SA. Development of an intracanal mature Enterococcus faecalis biofilm and its susceptibility to some antimicrobial intracanal medications; an in vitro study. Eur J Dent 2012;6:43-50.

7. Listgarten MA, Helldén L. Relative distribution of bacteria at clinically healthy and periodontally diseased sites in humans. J Clin Periodontol 1978;5:115-32.

8. Kuramitsu HK, He X, Lux R, Anderson MH, Shi W. Interspecies interactions within oral microbial communities. Microbiol Mol Biol Rev 2007;71:653-70.

9. Kelstrup J, Theilade J, Fejerskov O. Surface ultrastructure of some oral bacteria. Scand J Dent Res 1979;87:415-23.

10. Kigure T, Saito A, Seida K, Yamada S, Ishihara K, Okuda K. Distribution of porphyromonas gingivalis and treponema denticola in human subgingival plaque at different periodontal pocket depths examined by immunohistochemical methods. J Periodontal Res
1995;30:332-41.

11. Zijnge V, van Leeuwen MB, Degener JE, Abbas F, Thurnheer T, Gmür R, et al. Oral biofilm architecture on natural teeth. PLoS One 2010;5:9321.

12. Greif D, Wesner D, Regtmeier J, Anselmetti D. High resolution imaging of surface patterns of single bacterial cells. Ultramicroscopy 2010;110:1290-6.

13. Dubrovin EV, Voloshin AG, Kraevsky SV, Ignatyuk TE, Abramchuk SS, Yaminsky IV, et al. Atomic force microscopy investigation of phage infection of bacteria. Langmuir 2008;24:13068-74.

14. Allan EJ, Hoischen C, Gumpert J. Bacterial 1-forms. Adv Appl Microbiol 2009;68:1-39.

15. Bradley DE. Ultrastructure of bacteriophage and bacteriocins. Bacteriol Rev 1967;31:230-314.

16. MacDonald AB. Spirochetal cyst forms in neurodegenerative disorders: Hiding in plain sight. Med Hypotheses 2006;67:819-32.

17. Listgarten MA, Socransky SS. Electron microscopy of axial fibrils, outer envelope, and cell division of certain oral spirochetes. J Bacteriol 1964;88:1087-103.

18. De Ciccio A, McLaughlin R, Chan EC. Factors affecting the formation of spherical bodies in the spirochete treponema denticola. Oral Microbiol Immunol 1999;14:384-6.

19. Bik EM, Long CD, Armitage GC, Loomer P, Emerson J, Mongodin EF, et al. Bacterial diversity in the oral cavity of 10 healthy individuals. ISME J 2010;4:962-74.

20. Quevedo B, Giertsen E, Zijnge V, Lüthi-Schaller H, Guggenheim B, Thurnheer $\mathrm{T}$, et al. Phylogenetic group- and species-specific oligonucleotide probes for single-cell detection of lactic acid bacteria in oral biofilms. BMC Microbiol 2011;11:14

21. Dunne WM. Bacterial adhesion: Seen any good biofilms lately? Clin Microbiol Rev 2002,;15:155-66

22. Preus HR, Olsen I, Gjermo P. Bacteriophage infection--a possible mechanism for increased virulence of bacteria associated with rapidly destructive periodontitis. Acta Odontol Scand 1987;45:49-54.

23. Hansma HG, Pietrasanta L. Atomic Force Microscopy and other scanning probe microscopies. Curr Opin Chem Biol 1998;2:579-84.

\begin{tabular}{|l|l|}
\hline \multicolumn{2}{|c|}{ Access this article online } \\
\hline Quick Response Code: & Website: \\
\hline & www.eurjdent.com \\
\cline { 2 - 2 } & \\
\hline
\end{tabular}

